\title{
The Impact of Mandatory Reporting of Non-Serious Safety Reports to EudraVigilance on the Detection of Adverse Reactions
}

\author{
Gianmario Candore $^{1} \cdot$ Sebastian Monzon ${ }^{1}$ (D ) Jim Slattery ${ }^{1} \cdot$ Loris Piccolo $^{1} \cdot$ Rodrigo Postigo $^{1} \cdot$ Xavier Xurz $^{1}$. \\ Sabine Strauss ${ }^{2,3} \cdot$ Peter Arlett $^{1}$
}

Accepted: 1 November 2021 / Published online: 8 December 2021

(c) The Author(s) 2021

\begin{abstract}
Introduction and Objective European Union legislation has mandated the submission of European Economic Area nonserious reports to the EudraVigilance database since November 2017. As spontaneous reports of suspected adverse reactions to medicines represent a key source of safety signals, the European Medicines Agency has undertaken this work to assess the effects of this requirement on the characteristics of the reports submitted to EudraVigilance and on the detection of adverse drug reactions through routine analyses of the database.

Methods Changes in the numbers of serious and non-serious reports transmitted to EudraVigilance were examined over the period during which the legislation was implemented. The numbers and nature of potential safety signals emerging from established statistical algorithms used at the European Medicines Agency applied either to only the serious reports or to all reports in EudraVigilance were compared.

Results Up to November 2017, less than 25\% of European Economic Area reports in EudraVigilance were classified as non-serious, since than this figure was slightly above $60 \%$. This change accompanied an increase in the total number of reports received. Addition of non-serious reports to the signal detection process resulted in a small overall increase in signals of disproportionate reporting with some new signals of disproportionate reporting appearing and some existing signals of disproportionate reporting disappearing; the sensitivity of the signal detection system was slightly increased and the proportion of signals of disproportionate reporting that corresponded to known adverse drug reactions (a measure of efficiency) was unchanged.

Conclusions The change in legislation has led to a small increase in sensitivity, without affecting the efficiency of the routine statistical measures used. The number of non-serious reports as a proportion of reports in EudraVigilance is likely to increase over time and further monitoring of the impact on signal detection is required. Further work is also required on the qualitative impact of non-serious reports on the nature of signals detected and on their evaluation.
\end{abstract}

\section{Introduction}

The European Union pharmacovigilance legislation adopted in December $2010[1,2]$ brought about significant changes to reporting requirements for suspected adverse reactions

Gianmario Candore, Sebastian Monzon have shared first authorship.

Sebastian Monzon

sebastian.monzon@ema.europa.eu

1 European Medicines Agency, Domenico Scarlattilaan 6, 1083 HS Amsterdam, The Netherlands

2 CBG-MEB, Utrecht, The Netherlands

3 EMA Pharmacovigilance Risk Assessment Committee, Amsterdam, The Netherlands
[3]. An important change was the new requirement for Marketing Authorisation Holders and National Competent Authorities to report to EudraVigilance (EV) all non-serious cases originating from the European Economic Area (EEA). In addition, the new legislation brought an obligation on Marketing Authorisation Holders and National Competent Authorities to record and report cases received from patients, who cannot report directly to EV. In order to put into effect these changes, enhancements to the EV system were required and an audit demonstrating that the technical requirements were met had to take place. Following these steps, on 22 November, 2017, the enhanced EV was launched, accommodating the increased volumes of reports, supporting the use of international standards, including the International Council for Harmonisation (ICH) E2B(R3) on the data elements for transmission of individual case safety 


\section{Key Summary Points}

Up to and including November 2017, less than 25\% of European Economic Area reports in EudraVigilance were classified as non-serious, since then this figure raised to $61.6 \%$.

Comparison to a set of known adverse drug reactions suggested that including the non-serious reports slightly increased the sensitivity of the signal detection system (from 10.8 to $11.2 \%$ ) whereas the positive predictive value was unchanged (at 13.6\%).

Comparing the signal of disproportionate reporting appearing using only the serious reports and the signals of disproportionate reporting appearing using all reports (serious and non-serious) showed that $91.9 \%$ of signals of disproportionate reporting were unchanged. For the minority of signals of disproportionate reporting that changed, differences with respect to the profile of both adverse events and products were noticed; the impact of these changes, even if currently limited, might require further assessment in the future.

reports, and increasing access, analytical tools and transparency [4]. With these system enhancements, the new reporting requirements entered into force.

Signals of new safety issues with medicines, or a new aspect of a known issue, may originate from multiple sources, with spontaneous reports of suspected adverse reactions to medicines contributing to more signals than any other source [5]. EudraVigilance is the system for managing and analysing this information in the EEA. Therefore, when it comes to the overall performance of the EV system, a legitimate question is whether the mandatory reporting of non-serious reports (NSRs) from 22 November, 2017 has had an effect on the detection of safety signals when signal detection processes are conducted in all other respects according to the established process $[6,7]$.

This paper reports on a series of analyses performed to assess the increase and nature of the NSRs and their effect on signal detection in EV. The work is a contribution to the Pharmacovigilance Risk Assessment Committee Strategy on Measuring the Impact of Pharmacovigilance Activities [8], it is in line with the Pharmacovigilance Risk Assessment Committee implementing regulations of regularly reviewing the methodology(ies) used and publishing recommendations, and its results inform the pharmacovigilance process review and potential improvements.

\section{Methods}

The analyses were aimed at assessing the impact of NSRs on statistical routine drug safety monitoring at the European Medicines Agency (EMA) and hence the extraction of cases from EV followed the same default criteria applied when preparing the data for screening, which includes the calculation of signals of disproportionate reporting (SDRs) as described in section 4.1.5 of the guideline on 'Screening for adverse reactions in EudraVigilance' [7]. These reports are referred to as spontaneous in the rest of the article.

\subsection{Descriptive Analysis}

In order to assess the way the legislation has changed the reporting volumes, the trend of NSRs received in EV was examined across a period that included the implementation date of 22 November, 2017. The absolute number of all spontaneous reports (serious and non-serious) and of NSRs received in EV was calculated from 01/07/2012 to $30 / 06 / 2019$, as well as the proportion of NSRs relative to the total. Results were further broken down by cases arising in the EEA only. As the legislation only mandated the reporting of NSRs from the EEA, most analyses focused on these reports.

Changes in numbers and proportions of NSRs reported to $\mathrm{EV}$ were analysed to identify possible patterns according to patient age groups, primary source qualification and country of origin. A potential complicating factor in the analysis was the coincident introduction of the possibility to submit the cases in the new ICH-E2B(R3) data format that requires seriousness to be stipulated at a reaction level, whereas in ICH-E2B(R2) the seriousness is assigned at a case level (meaning that only the case could be categorised as serious or non-serious). To deal with both reporting standards in the analysis, the same logic used with ICH-E2B(R2) cases was applied to cases reported in ICH-E2B(R3) (i.e. if at least one reaction was reported as serious, the case was considered serious). Moreover, some analyses focusing on cases reported in ICH-E2B(R3) have been included to understand how seriousness at a reaction level is reported.

\subsection{Signals of Disproportionate Reporting}

This part of the study focused on estimating the impact of the mandatory reporting of NSRs on the statistical detection of safety signals. Several disproportionality measures have been proposed for signal detection purposes [8-11]; the statistical indicator of potential drug safety problems used routinely in EV is the reporting odds ratio (ROR) [7] 
(Table 1 of the Electronic Supplementary Material [ESM]). The ROR is calculated in each of five macro geographical regions of reporting (Europe, North America, Japan, rest of Asia and rest of the world [12]) and a SDR is raised for a specific drug-reaction combination if, in at least one macro region, all the following criteria are satisfied:

the lower bound of the $95 \%$ confidence interval of the ROR is greater than one;

the number of individual cases is greater than or equal to three for active substances contained in medicinal products included in the additional monitoring list [15] or five for the other active substances;

the event belongs to the Important Medical Events list [16], a subset that reflects more closely the concerns of pharmacovigilance experts.

For each of the analyses, the number and the character of SDRs arising from routine statistical signal detection methods performed by the EMA for substances included in Centrally Authorised Products (CAPs) were calculated and then compared for either (i) spontaneous serious reports only or (ii) all spontaneous reports (serious and non-serious).

\subsubsection{Comparing SDRs}

To assess whether including NSRs generated a different set of potential signals, and the magnitude of this change, SDRs raised with only the serious reports were compared to SDRs raised with all reports, and as a result three groups were identified: (i) SDRs raised in both scenarios; (ii) SDRs raised only from serious reports; and (iii) SDRs raised only from all reports (serious and non-serious).

\subsubsection{Performance Measures}

To compare the effectiveness of signal detection methods, a definition of the properties that constitute an effective signal detection system and a reference database to measure the extent to which these properties are achieved were required. Regarding the reference database, the publicly available PROTECT ADR database of labelled reactions for CAPs [13] that maps the wording in section 4.8 of the Summary of Product Characteristics to Preferred Terms (PTs) of the Medical Dictionary for Regulatory Activities (MedDRA ${ }^{\circledR}$ ) was used. As the reference database includes only CAPs, the analysis described in this section was restricted only to CAPs.

In line with previous work $[9,10,12]$, the measures chosen to compare the effectiveness of signal detection were sensitivity (the proportion of adverse drug reactions [ADRs] from the reference database that were flagged as SDRs) and positive predictive value (PPV, the proportion of SDRs that corresponds to ADRs present in the reference database, also called 'precision' and considered a measure of efficiency).

The SDRs identified from serious reports and the SDRs from all reports were then compared with the ADRs in the PROTECT ADR database to calculate sensitivity and PPV. Several sensitivity analyses were conducted to assess whether results were dependent on some specific settings in the reference database and/or on the data used to calculate SDRs:

Reference dataset restricted only to post-marketing ADRs: the PROTECT ADR database allows us to recognize the ADRs that were identified or confirmed during post-marketing. As EV is used to identify post-marketing ADRs, restricting the reference database to such events might serve as a useful comparison;

Maximise the proportion of NSRs on the total spontaneous reports: even if from 22 November, 2017 the proportion of NSRs showed a marked increase, their weight on the totality of cases in EV is still modest.

Restricting the analysis to include only data from the implementation of the new legislation allowed an estimation of the impact of NSRs when their relative proportion was at the highest and it simulated what could happen in a few years' time as the weight of NSRs increases. As the legislation only mandated the reporting of NSRs from the EEA, an additional analysis further restricted to include only reports from the EEA allowed us to focus on the region where the proportion of NSRs is even higher.

Use of the discontinued method to identify SDRs: before the implementation of the new guideline on 'Screening for adverse reactions in EudraVigilance' [7], the disproportionality measure was calculated on the totality of spontaneous reports in EV, without considering the split by macro region. Using this now-discontinued method was considered a helpful analysis as it spread the weight of NSRs clustered in the EEA.

All the analyses listed addressed the question of whether including NSRs in the set of reports where the disproportionality algorithm is applied had an effect on the number and characteristics of SDRs; an alternative analysis was to explore the use of NSRs separately, answering the question whether the set of NSRs by itself could generate additional SDRs to improve signal detection. In this approach, tested by Seabrooke et al. [12], reports in EV were divided into two subgroups, serious and NSRs, and the ROR was estimated within each subgroup. An SDR was considered to exist if the conditions for an SDR were met within either of the two subgroups. 
Fig. 1 Quarterly numbers of total spontaneous and nonserious spontaneous reports in EudraVigilance

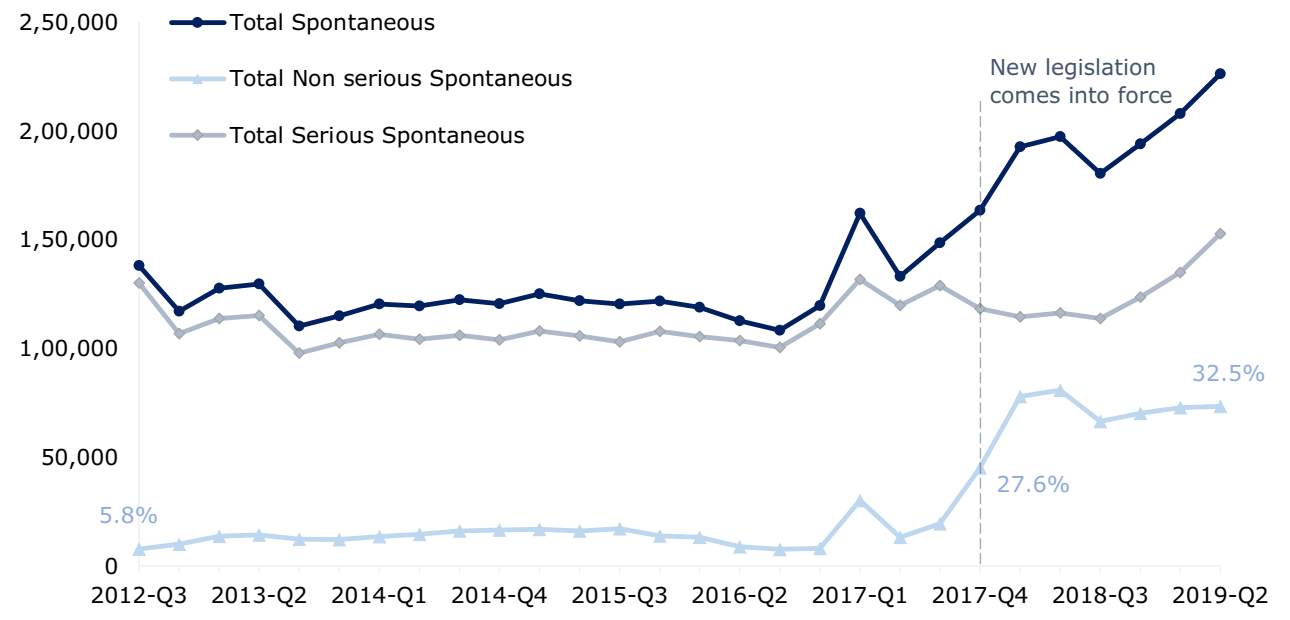

\subsubsection{Number of Reports per SDR}

As an additional measure to assess the potential impact on workload, the average and median number of reports associated with each SDR generated in the two scenarios (only serious reports or all reports) were compared.

\subsubsection{Individual Review of False Positives}

The underlying assumption of using a reference database of only positive controls is that any SDR with the event not coded in the reference is considered as a false positive rather than an unknown association [14]. The consequence is that the estimate of PPV obtained will be slightly conservative, but, more importantly for the scope of this analysis, any comparative evaluation between SDRs from only serious reports and from all reports is unlikely to be misleading as both scenarios should roughly be equally disadvantaged by inaccuracies in the list of positive controls.

To have some empirical confirmation of the false positives in both scenarios, 40 SDRs considered as false positive and appearing only in one scenario (20 SDRs raised only from serious reports and 20 SDRs raised only from all reports) were selected from those with the highest ROR and reviewed individually and independently by two experienced assessors in signal management to confirm they were not potential signals.

\subsubsection{Profiling of SDRs Appearing in Only One Scenario}

The profile of SDRs appearing only when the disproportionality algorithm was applied to serious reports and of SDRs appearing only when it was applied to all reports (serious and non-serious) were further analysed to investigate whether they differed in term of reactions and substances involved, and whether any pattern could be established.

\section{Results}

\subsection{Descriptive Analysis}

\subsubsection{Chronological Trend of NSRs in EV}

Figure 1 shows a marked increase of NSRs both in absolute number and as proportion of the total number of cases reported in EV. Figure 2 shows that the change was more pronounced when the EV analysis was restricted to EEA cases, where NSRs represent $61.6 \%$ of the reports submitted after the new reporting requirements on NSRs entered into force. Up to and including November 2017, less than $25 \%$ of EEA reports in EV were classified as non-serious, since then this figure raised to $61.6 \%$.

\subsubsection{Reporting by Primary Source: EEA}

Reports of suspected ADRs may be initiated by a patient, by a healthcare professional (HCP), or by both a patient and HCP. ${ }^{1}$ Litigation cases have not been considered here in line with the criteria described in section 4.1.5 of the guideline on 'Screening for adverse reaction in EudraVigilance' [7]. Table 1 shows the increases in the proportion of NSRs from each source following the legislation. A marked increase in the proportion of NSRs was seen in each of the three categories of primary source reporting with the reports originated by a patient remaining the category with the highest proportion of NSRs. The category of 'patient and HCP' saw

\footnotetext{
${ }^{1}$ An example of the latter is when a Marketing Authorisation Holder receives a report and then follows up with the reporter to obtain additional relevant information. Where the first reporter was a patient, the organisation performing the follow-up will usually ask for permission to contact their HCP to obtain such information. If the information is obtained, then there are both patient and HCP reporters for the same case.
} 
Fig. 2 Quarterly numbers of European Economic Area (EEA) total spontaneous and non-serious spontaneous reports in EudraVigilance

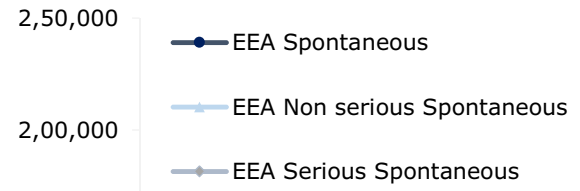

New legislation comes into force

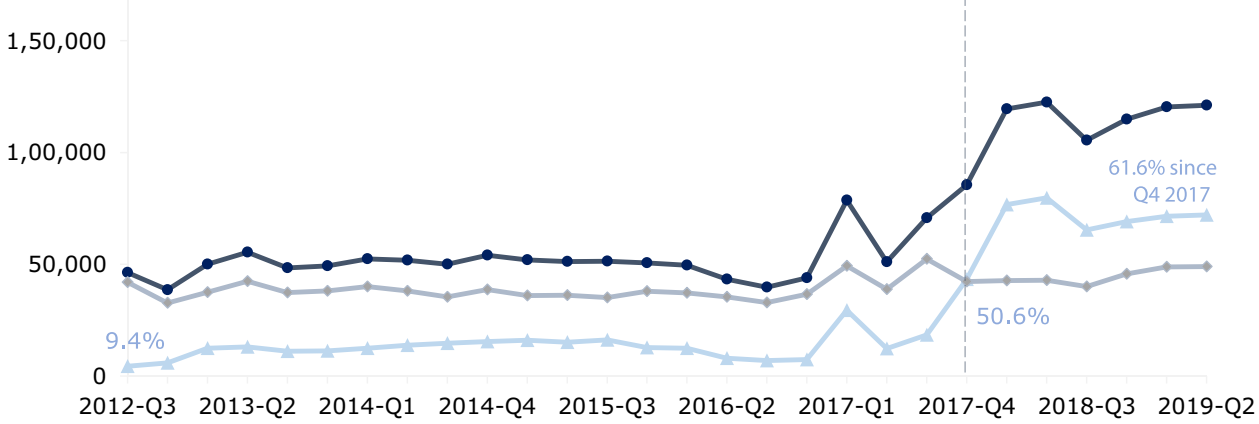

the biggest increase in relative terms. The category of 'only patient' also had the largest increase in absolute numbers of reports (serious and non-serious) in comparison with the levels before the implementation of the legislation.

\subsubsection{Reporting by Age of Subject: EEA}

There was some variation in increases in NSRs by age of the subject of the report (Table 2). The biggest increase in the relative proportion of NSRs was seen in the adult category and when age was not reported; paediatric remained the category with the highest proportion of NSRs among the categories with age reported.

\subsubsection{Reporting by Country: EEA}

Figure 3 shows that some countries were already sending high numbers of NSRs before the change in the reporting requirements, as EV permitted the voluntary submission of NSR before the implementation of the new legislation. However, after the change, most countries range around $\sim 60 \%$ of NSRs and the variation in rates between countries was much decreased (see Table 2 of the ESM).

\subsubsection{Impact of the New ICH-E2B(R3) Reporting Standard: EEA}

The ICH-E2B(R3) reporting standard requires seriousness to be assigned for each suspected reaction in the report, allowing serious and non-serious reactions to be individually identified in the same report. The left side of Table 3 shows the number and the type of adverse events for the cases reported in $\mathrm{EV}$ with the ICH-E2B(R3) format. The total number of reports received $(200,549)$ represented more than a quarter (26.4\%) of the total spontaneous cases received in EEA after the new legislation came into force. Case reports where both serious and non-serious reactions are reported represent a minority (approximately 8\%).

Thus, while there is a possibility that the advent of ICH-E2B(R3) could have qualitatively changed the nature of some reports-as non-serious adverse reactions under ICH-E2B(R2) could have previously been hidden under what is now a report with both serious and non-serious reactions-we consider this to be limited in number and therefore unlikely to have a large impact on this study. Finally, the right side of Table 3 also shows how reports with both serious and non-serious reactions have on average twice the number of adverse reactions compared with the reports with only either serious or only non-serious reactions.

\subsection{Impact on Statistical Signal Detection}

\subsubsection{Comparing SDRs}

The SDRs under the two scenarios (only serious or all reports) were compared; the majority $(83,170$, representing $91.9 \%$ of all SDRs) were unchanged, with only a small fraction of SDRs appearing in one scenario only, therefore no major changes were expected in the subsequent analyses. Including NSRs led to an overall increase of 5.5\% of SDRs, with 5981 new SDRs, but also 1337 that were lost (see Fig. 4).

A small increase in the number of SDRs appeared when NSRs were added to serious reports. However, it is not immediately clear whether this increase can be attributed to the reliability of information contained in the NSRs or to the increase in the volume of reports. This is because an increase in volume of reports can also impact on the number of SDRs $[9,10,12]$.

To investigate that the increase in volume would naturally increase the number of SDRs, a set of reports of the same number as the NSRs were created. To make this set not completely random, the same products and adverse events as 
Table 1 Number and proportion of non-serious reports before and after the reporting rule change, split by primary source

\begin{tabular}{|c|c|c|c|c|c|}
\hline Primary source & Period & Serious & Non- serious & $\begin{array}{l}\text { Total (serious and non- } \\
\text { serious) }\end{array}$ & $\%$ Non-serious \\
\hline \multirow[t]{2}{*}{ Only patient } & 1 July 2012-21 Nov 2017 & 117,193 & 57,374 & 174,567 & 32.9 \\
\hline & 22 Nov 2017-30 Jun 2019 & 45,635 & 172,204 & 217,839 & 79.1 \\
\hline \multirow[t]{2}{*}{ Patient + HCP } & 1 July 2012-21 Nov 2017 & 49,701 & 4994 & 54,695 & 9.1 \\
\hline & 22 Nov 2017-30 Jun 2019 & 17,047 & 19,793 & 36,840 & 53.7 \\
\hline \multirow[t]{2}{*}{ Only HCP } & 1 July 2012-21 Nov 2017 & 660,873 & 216,793 & 877,666 & 24.7 \\
\hline & 22 Nov 2017-30 Jun 2019 & 228,441 & 275,190 & 503,631 & 54.6 \\
\hline
\end{tabular}

$H C P$ healthcare professional

\section{Only Patient}

$9,00,000$

$7,50,000$

$6,00,000$

$4,50,000$

$3,00,000$

$1,50,000$

0

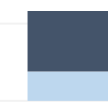

before

21/11/17

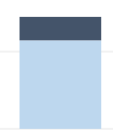

after
Patient + HCP

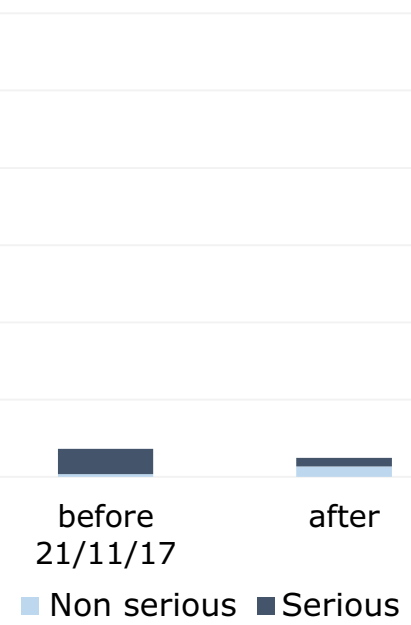

Non serious $\mathrm{Serious}$
Only HCP

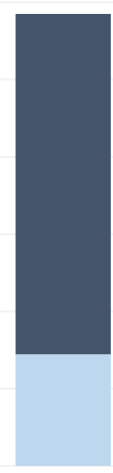

before

$21 / 11 / 17$

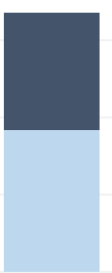

after the NSRs set were used, but their combination was assigned at random. Therefore, the set of random reports consisted exactly of the same number for each product and adverse event as in the NSRs, but the combinations between products and adverse events were different. When these reports, from which the original valid information had been replaced by random information, were used, an increase of $4.4 \%$ in SDRs was found, compared to $5.5 \%$ with true NSRs. These results were consistent over ten repetitions of the randomisation.

\subsubsection{Performance of Signal Detection: CAPs}

Review of the reference database revealed that many (81\%) of the ADRs at PT level listed in the Summary of Product Characteristics for the CAPs have never been reported in spontaneous reports to EV. This was unchanged by inclusion of NSRs (see Table 3 of the ESM).

In terms of performance, based on CAPs only, using both serious and NSRs resulted in a marginal increase in true and false positives compared to focusing only on serious reports. The results in Table 4 show a slightly higher sensitivity $(\sim 0.4 \%)$ and the same PPV when using both three and five as the threshold for the number of cases to define an SDR. These results were qualitatively unchanged in all the sensitivity analyses performed. See Table 5 for results using only adverse drug reactions identified postmarketing in the reference database. The alternative analysis to explore the use of NSRs separately revealed that the set of NSRs by itself could generate only a very limited number of additional ADRs (26) and this approach led to a slightly lower sensitivity and roughly the same PPV when compared with the main approach used in this study of analysing serious and NSRs together (see Tables 4 and 5 of the ESM).

To verify whether the impact on sensitivity and PPV can be mainly attributed to the reliability of information contained in the NSRs or to the increase in the volume of reports, the same set of random NSRs described in the previous paragraph was added. A small increase in sensitivity was still obtained but at the cost of a small decrease in PPV; using the five reports threshold, sensitivity rose from 10.8 to $10.9 \%$ while PPV decreased from 13.6 to $13.5 \%$. 
Table 2 Number and proportion of non-serious reports before and after the reporting rule change, split by age group

\begin{tabular}{|c|c|c|c|c|c|}
\hline Age group & Time & Serious & Non-serious & $\begin{array}{l}\text { Total (serious and non- } \\
\text { serious) }\end{array}$ & $\%$ Non-serious \\
\hline \multirow[t]{2}{*}{ Paediatric } & 1 July 2012-21 Nov 2017 & 71,618 & 49,562 & 121,180 & 40.9 \\
\hline & 22 Nov 2017-30 Jun 2019 & 25,932 & 41,129 & 67,061 & 61.3 \\
\hline \multirow[t]{2}{*}{ Adult } & 1 July 2012-21 Nov 2017 & 360,398 & 123,461 & 483,859 & 25.5 \\
\hline & 22 Nov 2017-30 Jun 2019 & 124,585 & 188,253 & 312,838 & 60.2 \\
\hline \multirow[t]{2}{*}{ Geriatric } & 1 July 2012-21 Nov 2017 & 276,756 & 79,055 & 355,811 & 22.2 \\
\hline & 22 Nov 2017-30 Jun 2019 & 95,405 & 108,426 & 203,831 & 53.2 \\
\hline \multirow[t]{2}{*}{ Missing } & 1 July 2012-21 Nov 2017 & 120,453 & 27,083 & 147,536 & 18.4 \\
\hline & 22 Nov 2017-30 Jun 2019 & 45,201 & 129,379 & 174,580 & 74.1 \\
\hline
\end{tabular}

Paediatric

Adult

$5,00,000$

$4,00,000$

$3,00,000$

$2,00,000$

$1,00,000$

0

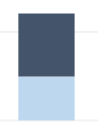

before after 21/11/17

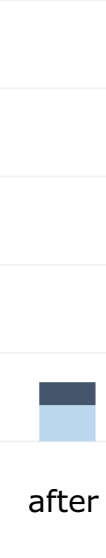

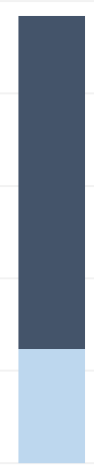

before 21/11/17

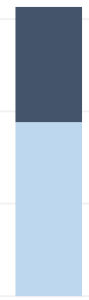

after
Geriatric

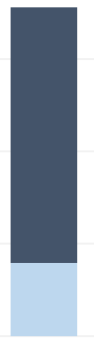

before 21/11/17

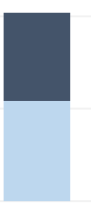

after

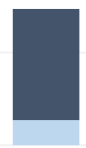

before 21/11/17
Missing Age

Non serious $\square$ Serious

These results were consistent over ten repetitions of the randomization.

\subsubsection{Number of Reports per SDR}

Including NSRs generated 5.5\% more SDRs; however, the average number of reports (individual case safety reports) associated with each SDR was similar to the average when only serious reports were considered, at just below 40, and the median was unchanged at nine in both scenarios.

\subsubsection{Individual Review of False Positives}

The result of the individual and independent review by two signal management assessors of 20 SDRs unique to each scenario, i.e. from only serious or from all spontaneous reports, not in the reference database did not identify any potential signals for further review.

\subsubsection{Profiling of SDRs Appearing in Only One Scenario}

The unique SDRs generated analysing only the serious reports were contrasted with those unique SDRs generated analysing serious and NSRs. Table 6 shows the MedDRA ${ }^{\circledR}$ PTs most reported in these discordant sets of SDRs.

On each side of the table, the PTs are ranked by frequency in the discordant set in the heading, but the 'Rank' column indicates the ordering of that particular PT amongst SDRs generated by the other report set. It can be seen that there were some unexpected changes in the SDRs found when NSRs were added, such as the serious disorder of Stevens-Johnson syndrome making it to the top place as well as other serious conditions (e.g. circulatory collapse or multiple organ dysfunction syndrome).

Emphasis of statistical signal detection might refocus on to different clinical domains with the inclusion of NSRs. An indication of such a change can be obtained by examining the relative risk that an SDR will be generated in each 


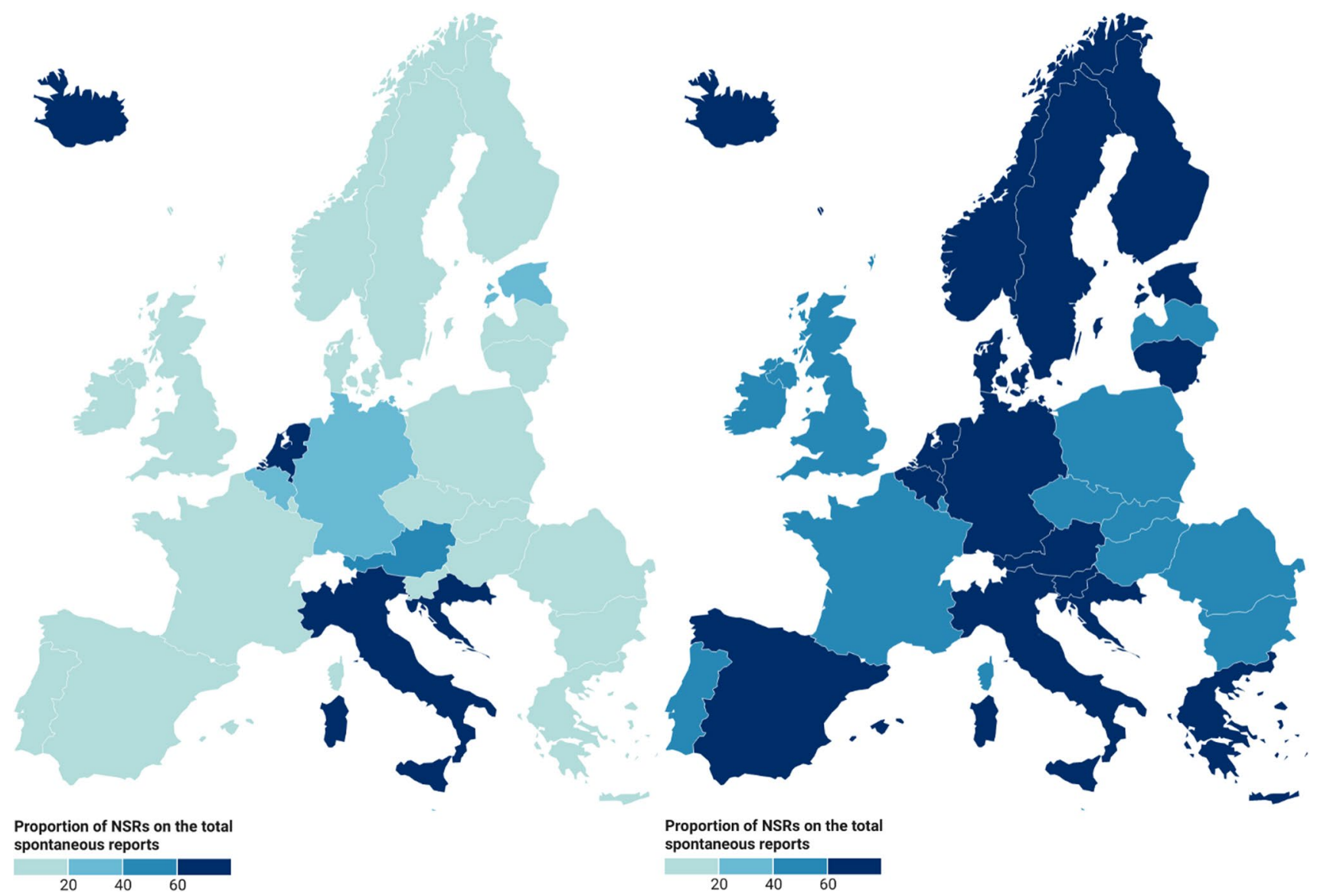

Fig. 3 Proportion of non-serious reports (NSRs) on the total spontaneous reports before and after the report rule change, split by European Economic Area countries (As the data for this analysis were

retrieved prior to its withdrawal from the European Union, the UK has been considered as part of the EEA.)
Table 3 Total number of reports and mean number of adverse events per report submitted in ICH-E2B(R3)

\begin{tabular}{lcll}
\hline Type of report & $\begin{array}{l}\text { No. of reports in ICH- } \\
\text { E2B(R3) format }\end{array}$ & $\begin{array}{l}\text { Non-serious adverse reac- } \\
\text { tions per report (mean) }\end{array}$ & $\begin{array}{l}\text { Serious adverse } \\
\text { reactions per report } \\
\text { (mean) }\end{array}$ \\
\hline Only serious & 65,869 & Not applicable & 2.8 \\
Serious and non-serious & 16,416 & 2.5 & 2.8 \\
Only non-serious & 118,264 & 2.4 & Not applicable \\
\hline
\end{tabular}

ICH International Council for Harmonisation
MedDRA ${ }^{\circledR}$ System Organ Class when NSRs are included compared with using only serious reports. Figure 5 shows the System Organ Classes ordered by this relative risk. For example, the risk that an SDR will be occurring in the 'Reproductive system and breast disorders' category is raised by a factor of 1.135 when NSRs are included.

The SDRs that differ when contrasting the analyses of only serious reports vs serious reports and NSRs may also change the focus with respect to substances to be investigated. Table 7 shows that there is no overlap in the most frequently reported substances in the discordant SDRs. The display of frequency and ranking order follows the same rationale as in Table 6.

\section{Discussion}

The change in the rules for reporting suspected ADRs to EV came into effect in November 2017 and has resulted in an increased level of reporting of NSRs, both in absolute number and in relation to the total. The change was more pronounced when the analysis was restricted to cases 
Fig. 4 Signals of disproportionate reporting (SDRs) considering only serious or all spontaneous reports in EudraVigilance (threshold of at least five cases applied). NSRs non-serious reports

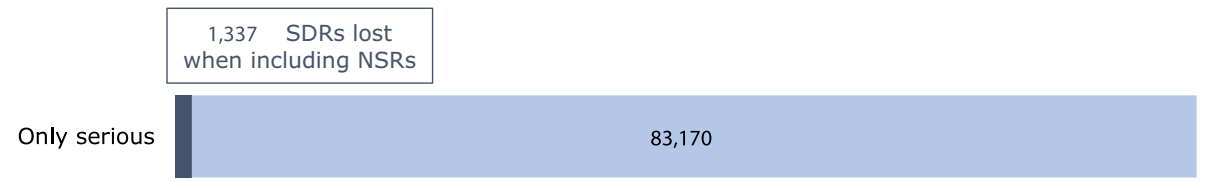


Table 5 Performance of signal detection methods on Centrally Authorised Products: sensitivity analysis using only adverse drug reactions identified postmarketing in the reference database

\begin{tabular}{llllll}
\hline Methods & Total SDRs & True positive & False positive & Sensitivity & PPV \\
\hline ROR_n3: only serious & 24,311 & 741 & 23,570 & $27.5 \%$ & $3.0 \%$ \\
ROR_n3: including NSR & 25,012 & 757 & 24,255 & $28.0 \%$ & $3.0 \%$ \\
Change (\%) & $+2.9 \%$ & $+2.2 \%$ & $+2.9 \%$ & & \\
ROR_n5: only serious & 16,083 & 682 & 15,401 & $25.3 \%$ & $4.2 \%$ \\
ROR_n5: including NSR & 16,523 & 693 & 15,830 & $25.7 \%$ & $4.2 \%$ \\
Change (\%) & $+2.7 \%$ & $+1.6 \%$ & $+2.8 \%$ & & \\
\hline
\end{tabular}

NSR non-serious report, $P P V$ positive predictive value, $R O R$ reporting odds ratio, SDRs signals of disproportionate reporting

Table 6 Top 12 MedDRA $^{\circledR}$ PTs most reported in the discordant sets of signal of disproportionate reporting

\begin{tabular}{|c|c|c|c|c|c|}
\hline \multicolumn{3}{|l|}{ Only serious (1337 SDRs) } & \multicolumn{3}{|l|}{ Serious and non-serious (5981 SDRs) } \\
\hline PT code & No. of SDRs & $\begin{array}{l}\text { Rank (in serious } \\
\text { and non-serious) }\end{array}$ & PT Code & No. of SDRs & $\begin{array}{l}\text { Rank (in } \\
\text { only seri- } \\
\text { ous) }\end{array}$ \\
\hline Anaphylactic shock & 35 & 44 & Stevens-Johnson syndrome & 73 & 145 \\
\hline Angioedema & 30 & 19 & Seizure & 61 & 42 \\
\hline Loss of consciousness & 30 & 5 & Erectile dysfunction & 58 & 15 \\
\hline Syncope & 27 & 27 & Circulatory collapse & 58 & 16 \\
\hline Polyneuropathy & 21 & 67 & Loss of consciousness & 55 & 2 \\
\hline Anaphylactic reaction & 18 & 33 & Thrombocytopenia & 55 & 26 \\
\hline Hemiparesis & 16 & 104 & Respiratory distress & 53 & 9 \\
\hline Hepatitis & 15 & 28 & Death & 53 & 20 \\
\hline Multiple sclerosis & 14 & 637 & Pneumonia & 48 & 9 \\
\hline Depressed level of consciousness & 14 & 48 & Arrhythmia & 47 & 16 \\
\hline Pneumonia & 14 & 9 & Renal failure & 46 & 31 \\
\hline Respiratory distress & 14 & 7 & Multiple organ dysfunction syndrome & 46 & 31 \\
\hline
\end{tabular}

MedDRA ${ }^{\circledR}$ PTs common to the two sets of discordant SDRs are in italic

MedDRA ${ }^{\circledR}$ Medical Dictionary for Regulatory Activities, PTs Preferred Terms, SDRs signals of disproportionate reporting

Fig. 5 Number and relative risk of signals of disproportionate reporting by Medical Dictionary for Regulatory Activities (MedDRA $^{\circledR}$ ) System Organ Class when non-serious reports are included

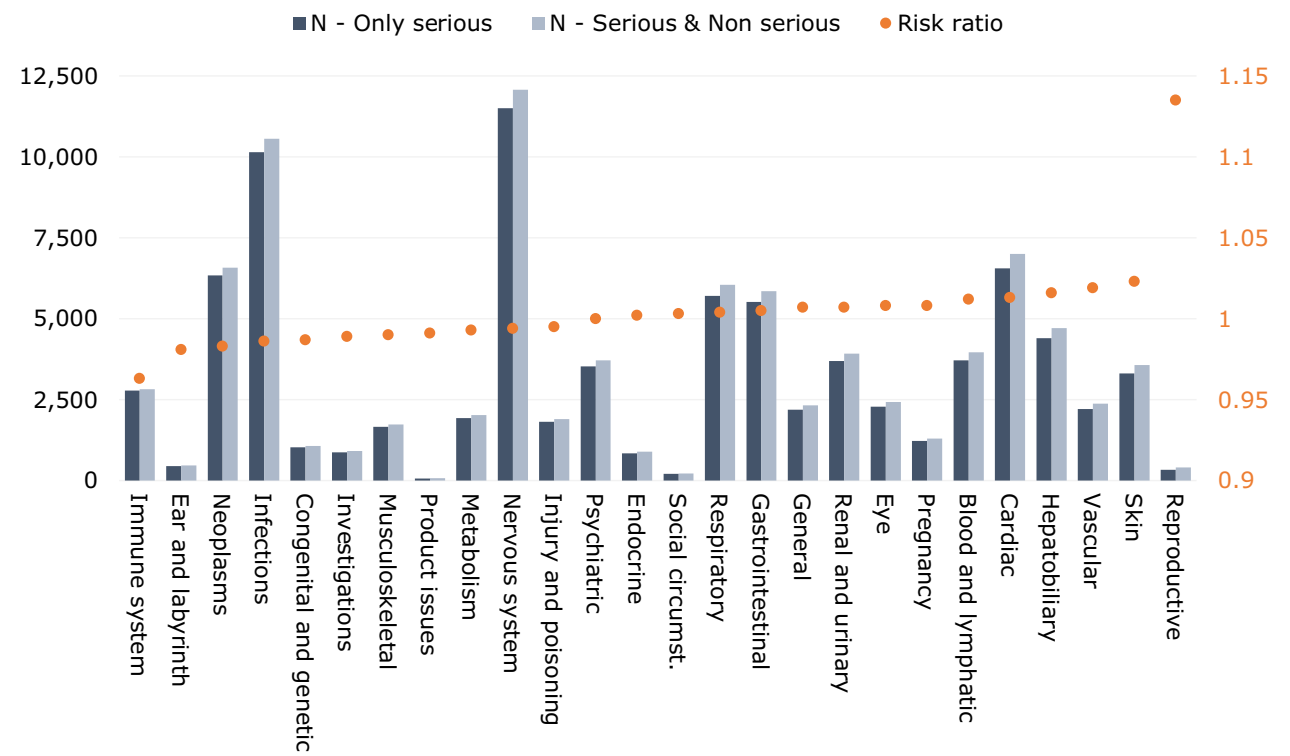


Table 7 Top ten most reported substances in the discordant sets of SDRs

\begin{tabular}{|c|c|c|c|c|c|}
\hline \multicolumn{3}{|l|}{ Only serious (1337 SDRs) } & \multicolumn{3}{|c|}{ Serious and non-serious (5981 SDRs) } \\
\hline Active substance & No. of SDRs & $\begin{array}{l}\text { Rank (in serious } \\
\text { and non-serious) }\end{array}$ & Active substance & No. of SDRs & $\begin{array}{l}\text { Rank (in } \\
\text { only seri- } \\
\text { ous) }\end{array}$ \\
\hline Etanercept & 148 & 180 & Fluconazole & 56 & 78 \\
\hline Pregabalin & 43 & 905 & Troglitazone & 43 & 123 \\
\hline Varenicline & 40 & 905 & Prednisolone & 37 & No SDRs \\
\hline $\begin{array}{l}\text { Human papillomavirus vaccine [types } 6,11,16,18] \text { (recom- } \\
\text { binant, adsorbed) }\end{array}$ & 28 & 57 & Adalimumab & 36 & No SDRs \\
\hline Sildenafil & 28 & 57 & Prednisone & 35 & No SDRs \\
\hline Varicella vaccine (live) & 25 & 318 & Cyclophosphamide & 33 & No SDRs \\
\hline Sitagliptin & 22 & 631 & Trovafloxacin & 32 & No SDRs \\
\hline Pneumococcal polysaccharide conjugate vaccine (adsorbed) & 21 & 497 & Ciclosporin & 32 & 192 \\
\hline Telmisartan & 18 & 631 & Rituximab & 32 & No SDRs \\
\hline Dabigatran & 18 & 180 & Azithromycin & 31 & 45 \\
\hline
\end{tabular}

SDRs signals of disproportionate reporting

arising in EEA, as expected because the relevant reporting rule change only applied to EEA reporting. The increase in the number of NSRs had also been affected by the new legislation mandating the submission of patients and consumer reports to $\mathrm{EV}$.

We have examined both the number and the character of SDRs arising from routine EMA statistical signal detection methods applied either solely to spontaneous serious reports or to all spontaneous reports, i.e. including the NSRs. The vast majority of SDRs (91.9\%) were common to both scenarios.

Inclusion of NSRs produced some new SDRs and eliminated others. Overall, an increase of about $5.5 \%$ of SDRs was noted; however, inclusion of a similar number of computer-generated reports, obtained by randomising the adverse event and substance lists from the NSRs, also consistently increased SDRs by $4.4 \%$. Although this demonstrates that the increase in SDRs that can be attributed to the information contained in the NSRs is only about $1.1 \%$, the increase in sensitivity when using randomised reports is only modest $(0.1 \%)$ and counterbalanced by a corresponding decrease in PPV. While when using the real NSRs, the increase in sensitivity raised from 10.8 to $11.2 \%$ and the PPV remained unchanged.

The maintenance of the PPV and consequently of efficiency in terms of SDRs per ADR detected is a positive finding and is reinforced by the finding that the average number of reports contributing to each SDR did not increase. Hence, the workload per ADR seems likely to have remained unchanged (even if the workload has increased overall). The different sensitivity analyses performed, looking at different time periods and/or focusing only on the set of ADRs identified post-marketing, showed the same pattern of slightly increased sensitivity and similar PPV, thus providing confidence on the robustness of the aggregated results.

The discordant SDRs highlighted only in one scenario are a minority ( $8.1 \%$ of the total SDRs); nevertheless, there were changes in the character of SDRs arising from reports that include NSRs. Both the nature of the adverse events and the sets of products have changed. However, the significance of these changes seems small when compared to the totality of the SDRs and the impact will only become clear in the long term as the proportion of NSRs in the database increases.

An interesting observation from the analysis was that there was considerable variation in transmission of NSRs between Member States prior to the legislation and some were already sending a high proportion if not all of their NSRs. Hence, an analysis of the potential impact of legislative changes could have been made prior to implementation and might have given useful information in shaping the legislation. The variation in transmission of NSRs between Member States decreased markedly after the legislation; the reasons for this remaining variation were not explicitly explored but likely reflect different cultures for reporting, different modalities being put in place by national competent authorities and different levels of promotion of patient reporting.

The analyses performed have some limitations. The timing to detect an ADR was not studied; although time is considered a key criterion for signal detection methods, the $91.9 \%$ overlap of SDRs between the two scenarios considered did not suggest an important change on any of the dimensions studied. The greater number of reports when NSRs are included would indicate that, if any effect on timing to detect an ADR was present, it would be on reaching 
more quickly the threshold on the number of cases to have an SDR.

Another limitation is that the reference database was based only on CAPs; however, it is not expected that Nationally Authorised Products (NAPs) would systematically differ from CAPs in term of performance of statistical signal detection. The analysis showing that $91.9 \%$ of SDRs remained unchanged included NAPs and reinforces the suggestion that the conclusions would not be impacted.

Finally, the analysis of the effect of including NSRs was mainly limited to the impact on statistical signal detection; no attempt was made to establish whether there is any effect on subsequent phases of signal management including signal evaluation. Potential areas of further research could include determining whether NSRs provide more evidence to decide whether to validate, or not, a signal, or whether the proportion of NSRs on the total number of reports for each SDR could be used as an additional indicator to prioritise, or not, the drug-reaction combination for review.

\section{Conclusions}

The November 2017 change in reporting rules to EV mandating submission of NSRs of suspected ADRs originating in the EEA has resulted in a substantial increase in the number of such reports received. This change has led to a small increase in the sensitivity of the routine statistical signal detection used to detect safety signals at the EMA and has not affected its efficiency. The number of SDRs per ADR detected and the median number of reports contributing to each SDR have remained constant.

Some new SDRs were detected when NSRs were included in the calculations and some SDRs were eliminated. The characteristics of these discordant SDRs were different both with respect to the profile of the adverse event and with respect to the products. These changes, even if impacting only a small proportion of SDRs, require long-term monitoring and careful scientific evaluation to understand whether there is any impact on the type of statistical signal detected and any implications for methods and processes used. However, at the current time and based on these results, it is not considered necessary to amend the current EMA practices in screening EV. The total proportion of NSRs is likely to increase over time and further monitoring of their impact will be required.

Supplementary Information The online version contains supplementary material available at https://doi.org/10.1007/s40264-021-01137-0.

Acknowledgements The authors thank Tom Paternoster-Howe, Gilles Touraille and Luis Pinheiro for their constructive review and comments.

\section{Declarations}

Funding Not applicable.

Conflicts of interest/Competing interests Not applicable.

Ethics approval Not applicable.

Consent to participate Not applicable.

Consent for publication Not applicable.

Availability of data and material Not applicable.

Code availability Not applicable.

Author contributions All authors contributed towards the writing of the text and have read and approved the final version.

Open Access This article is licensed under a Creative Commons Attribution-NonCommercial 4.0 International License, which permits any non-commercial use, sharing, adaptation, distribution and reproduction in any medium or format, as long as you give appropriate credit to the original author(s) and the source, provide a link to the Creative Commons licence, and indicate if changes were made. The images or other third party material in this article are included in the article's Creative Commons licence, unless indicated otherwise in a credit line to the material. If material is not included in the article's Creative Commons licence and your intended use is not permitted by statutory regulation or exceeds the permitted use, you will need to obtain permission directly from the copyright holder. To view a copy of this licence, visit http://creativecommons.org/licenses/by-nc/4.0/.

\section{References}

1. Directive 2010/84/EU.

2. Regulation (EU) No. 1235/2010.

3. Santoro A, Genov G, Spooner A, Raine J, Arlett P. Promoting and protecting public health: how the European Union pharmacovigilance system works. Drug Saf. 2017;40(10):855-69. https://doi. org/10.1007/s40264-017-0572-8.

4. Postigo R, Brosch S, Slattery J, et al. EudraVigilance Medicines Safety Database: publicly accessible data for research and public health protection. Drug Saf. 2018;41(7):665-75. https://doi.org/ 10.1007/s40264-018-0647-1.

5. Potts J, Genov G, Segec A, Raine J, Straus S, Arlett P. Improving the safety of medicines in the European Union: from signals to action. Clin Pharmacol Ther. 2020;107:521-9. https://doi.org/10. 1002/cpt.1678.

6. European Medicines Agency. Guideline on good pharmacovigilance practices (GVP). Module IX: signal management (Rev 1). https://www.ema.europa.eu/documents/scientific-guideline/guide line-good-pharmacovigilance-practices-gvp-module-ix-signalmanagement-rev-1_en.pdf. Accessed 10 Nov 2021.

7. European Medicines Agency. Screening for adverse reactions in EudraVigilance. https://www.ema.europa.eu/documents/other/ screening-adverse-reactions-eudravigilance_en.pdf. Accessed 10 Nov 2021.

8. European Medicines Agency. PRAC strategy on measuring the impact of pharmacovigilance activities (Rev 1). http://www.ema. europa.eu/docs/en_GB/document_library/Other/2016/01/WC500 199756.pdf. Accessed 10 Nov 2021. 
9. Candore G, Juhlin K, Manlik K, Thakrar B, Quarcoo N, Seabroke $\mathrm{S}$, et al. Comparison of statistical signal detection methods within and across spontaneous reporting databases. Drug Saf. 2015;38(6):577-87.

10. Wisniewski A, Bate A, Bousquet C, Brueckner A, Candore G, Juhlin K, et al. Good signal detection practices: evidence from IMI PROTECT. Drug Saf. 2016;39:469-90.

11. Van Puijenbroek EP, Bate A, Leufkens HGM, Lindquist M, Orre R, Egberts ACG. A comparison of measures of disproportionality for signal detection in spontaneous reporting systems for adverse drug reactions. Pharmacoepidemiol Drug Saf. 2002;11(1):3-10.

12. Seabroke S, Candore G, Juhlin K, Quarcoo N, Wisniewski A, Arani R, et al. Performance of stratified and subgrouped disproportionality analyses in spontaneous databases. Drug Saf. 2016;39(4):355-64.
13. PROTECT. Adverse drug reactions database. http://www.imiprotect.eu/adverseDrugReactions.shtml. Accessed 10 Nov 2021.

14. Slattery J. Measuring signal detection performance: can we trust negative controls and do we need them? Drug Saf. 2016;39:371-3. https://doi.org/10.1007/s40264-016-0407-z.

15. European Medicines Agency. Medicines under additional monitoring. https://www.ema.europa.eu/en/human-regulatory/postauthorisation/pharmacovigilance/medicines-under-additionalmonitoring. Accessed 10 Nov 2021.

16. EudraVigilance Expert Working Group. Inclusion/exclusion criteria for the "Important Medical Events". https://www.ema.europa. eu/en/documents/other/eudravigilance-inclusion/exclusion-crite ria-important-medical-events-list_en.pdf. Accessed 10 Nov 2021. 